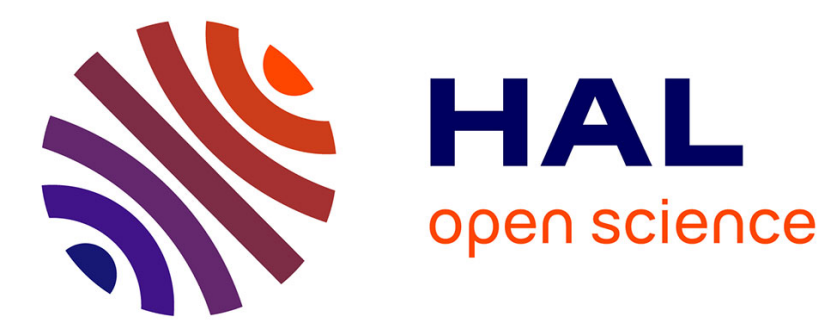

\title{
A l'origine des contradictions sur la villégiature
}

Xavier Lafon

\section{To cite this version:}

Xavier Lafon. A l'origine des contradictions sur la villégiature: Les villas de l'aristocratie romaine

(Ier siècle avant J.-C./Ier siècle aprés J.-C. . Histoire urbaine, 2014, 3 (41), pp.11-22. hal-01473712

\section{HAL Id: hal-01473712 \\ https://hal-amu.archives-ouvertes.fr/hal-01473712}

Submitted on 22 Feb 2017

HAL is a multi-disciplinary open access archive for the deposit and dissemination of scientific research documents, whether they are published or not. The documents may come from teaching and research institutions in France or abroad, or from public or private research centers.
L'archive ouverte pluridisciplinaire HAL, est destinée au dépôt et à la diffusion de documents scientifiques de niveau recherche, publiés ou non, émanant des établissements d'enseignement et de recherche français ou étrangers, des laboratoires publics ou privés. 


\title{
À l'origine des contradictions sur la villégiature
}

\author{
Les villas de l'aristocratie romaine \\ ( $\mathrm{I}^{\mathrm{er}}$ siècle avant J.-C. / $\mathrm{I}^{\mathrm{er}}$ siècle après J.-C.)
}

T e mot de villa apparaît assez tôt dans la langue latine puisque l'on connaît une villa publica dès le $\mathrm{v}^{\mathrm{e}}$ siècle avant J.-C. ${ }^{1}$ Il est encore présent dans la plupart des langues européennes, romanes ou non. Mais au-delà de cette apparente continuité, la notion même de villa a fortement évolué, tout en recouvrant, à quasiment toutes les étapes de son existence, des réalités contrastées, y compris déjà dans le monde romain lui-même ${ }^{2}$. Il n'est pas possible dans l'espace limité de cet article de reprendre le détail de cette histoire complexe, en particulier son renouveau au début de l'époque moderne, d'autres l'ont fait de façon exemplaire ${ }^{3}$. En revanche, il semble possible d'examiner, à travers les contradictions dans lesquelles se développe le concept comme la réalité de la villa romaine, un certain nombre de caractéristiques qui se sont maintenues dans les villas d'époques moderne et contemporaine. La démarche suivie ${ }^{4}$ sera autant historiogra-

\footnotetext{
* Aix Marseille Université, CNRS, Université Lyon 2, Université de Pau et des Pays de l'Adour, IRAA, USR 3155, 13284, Aix-en-Provence, France.

1. Tite Live, Histoire romaine, IV, 22, 7.

2. La bibliographie sur la création et l'évolution de la villa romaine est considérable! On peut retenir, pour des éclairages très différents, Andrea Carandini, «La villa romana e la piantagione schiavistica» dans Emilio Gabba et Aldo Schiavone (a cura di), Storia di Roma, 4, Caratteri e morfologie, Torino, Einaudi, 1986, p. 101-200; Harald Mielsch, Die Römische Villa. Architektur und Lebensform, München, C. H. Beck, 1987; Pierre Gros, L'architecture romaine, 2, Maisons, palais, villas et tombeaux, Paris, Picard (Les manuels d'art et d'archéologie antique), 2001.

3. Pour ne citer qu'un exemple, l'ouvrage de James S. Ackerman paru en anglais en 1990 (The villa, Form and Ideology of Country Houses), traduit aussi bien en italien (1992: La villa-Forma e ideologia) qu'en français (1997: La villa, de la Rome antique à Le Corbusier).

4. Je me permets de renvoyer pour plus de précision dans la démonstration à Xavier Lafon, Villa maritima, Recherches sur la villa littorale de l'Italie romaine, (III ${ }^{e}$ siècle avant-III ${ }^{e}$ siècle après J.-C.), Rome, EFR, 2001, (BEFAR 307), passim.
} 
phique que proprement historique, en raison de la prégnance de l'idéologie dans tout discours consacré à la villa de l'Antiquité jusqu'à nos jours.

La première discussion que nous connaissons consacrée au sens à donner au concept de villa figure dans le premier chapitre du livre III de l'Économie rurale (Res Rusticae) de Varron, publié en 37 av. J.-C ${ }^{5}$. Cette date est très postérieure à la naissance de la villa comme réalité sociale et architecturale mais également très tardive par rapport aux transformations qu'elle connait à partir du $\mathrm{II}^{\mathrm{e}}$ siècle, transformations décrites par Caton l'Ancien dans le De Agricultura publié avant 149 av. J.-C., date de sa mort. Ce décalage, dans un contexte où la nouveauté n'est pas considérée comme un progrès, favorise la mise en place d'une vision nostalgique du passé qui va rester attachée à la notion de villa bien au-delà de la période antique comme nous aurons l'occasion de le préciser ultérieurement. La forme du "dialogue aristotélicien» adoptée par Varron lui interdit de donner des définitions claires et univoques mais un certain nombre de constantes, plus exactement quelques points qui font l'unanimité, se dégagent de ce texte. La première, qui aura son importance encore à l'époque moderne, concerne l'opposition entre fonctions économiques et fonctions résidentielles. Le moraliste qu'est Varron regrette bien évidemment que les premières aient été amoindries au profit d'un développement exagéré des secondes mais, dans le dialogue instauré entre les protagonistes, personne ne réfute véritablement l'appellation de villas donnée à un ensemble de bâtiments purement résidentiels, du moment qu'ils sont construits hors les murs de la ville. En revanche, de façon certainement provocatrice, l'aspect résidentiel ne repose pas nécessairement sur le luxe des matériaux et du mobilier, œuvres d'art comprises, mais il ne l'exclut pas pour autant.

L'intérêt longtemps porté exclusivement aux œuvres d'art par les archéologues (sous la pression des collectionneurs) a conduit à négliger totalement les traces des activités économiques dans les restes conservés de villas romaines, alors même que les premières villas palladiennes étaient fondées de façon plus ou moins explicite sur la reconquête de terrains agricoles ${ }^{6}$. De fait, on voit s'établir chez Varron un clivage désormais général dans le monde romain entre des villas où la fonction productrice demeure essentielle (villae rusticae) et celles où la fonction résidentielle l'emporte (villae urbanae). En théorie cependant, la

5. Jacques Heurgon, Introduction au volume 1 des Res Rusticae, Paris, Belles-Lettres (CUF), 1978, p. XXI.

6. Richard. Bentmann et Michaël Müller, La villa, architecture de domination (traduction de l'allemand: Die Villa als Herrschaftsarchitektur, Frankfurt am Main, Shurkamp, 1971), Bruxelles, Mardaga, 1975, particulièrement p. 15-29. 
fonction productive demeure un élément clé, conduisant certains auteurs comme Pline le Jeune à des acrobaties littéraires pour justifier le caractère politiquement correct de certaines au moins de ses possessions : c'est ainsi qu'il parle de fructus (production) dans sa villa des Laurentes (près d'Ostie) à propos de sa "production » d'ouvrages littéraires qu'il peut y écrire? ${ }^{7}$.

La dissociation physique des lieux de production et de stockage de la résidence du propriétaire devient la règle dans les plus grands complexes italiens, ceux sur lesquels je porterai désormais exclusivement mon attention. Il s'agit d'une catégorie nécessairement limitée d'édifices, mais elle présente l'avantage d'avoir été bien traitée dans les textes des auteurs antiques, souvent propriétaires de tels édifices. La connaissance de ces auteurs constitue l'accès le plus facile à ces monuments et à la vie sociale qu'ils rendent possible, indépendamment de la connaissance des monuments eux-mêmes. Pour bien comprendre, malgré tout, le côté limité de l'échantillonnage retenu, il suffit de rappeler que des solutions différentes ont pu être préférées dans d'autres régions de l'empire comme suffit à le montrer, pour la question du rapport résidence/production, le cas des grandes villas des Trois Gaules: sans faire totalement exception à la règle de la séparation (la partie résidentielle dispose d'une enceinte particulière qui l'isole des autres bâtiments consacrés à la production), l'organisation générale du domaine tend à mettre en valeur sa richesse économique en disposant les bâtiments d'exploitation le long de l'accès principal à la villa: tout visiteur ne peut donc en ignorer l'importance. En Italie, au contraire, cette partie productive est davantage cachée à la vue du visiteur, phénomène que l'on retrouve dans la littérature impériale consacrée aux villas, malgré le poids idéologique que représente, comme on l'a vu, la "production» dans la littérature antérieure, notamment celle des agronomes comme Caton et Varron. L'exemple le plus caractéristique de cette attitude est offert par Pline le Jeune dans sa Correspondance. Comme propriétaire $^{8}$, soucieux de défendre ses intérêts économiques, la gestion de ses domaines agricoles est une question récurrente. Mais dans la description détaillée qu'il réserve à deux de ses villas où il réside de façon préférentielle, il n'est fait référence qu'à la seule partie résidentielle, sans même prendre en compte les espaces de service (cuisine, logements des esclaves) à l'exception de la simple mention d'un horreum dans une

7. Pline le Jeune, Lettres, II, 17.

8. Pline le Jeune, Lettres, IX, 37 (il ne peut assister à l'inauguration de la magistrature de son correspondant pour régler des contrats avec ses fermiers) ou III, 19 où il envisage d'acheter une propriété enclavée dans celle qu'il possède déjà pour rentabiliser les investissements. Cf. MosesI. Finley, L'économie antique, trad. fr., Paris, Éditions de Minuit, 1975, p. 149-150. 
tour ${ }^{9}$. C'est cette vision tronquée de la villa que retiendront les Modernes pour lesquels ces descriptions ont constitué le moyen d'accès privilégié à la "villa romaine ", à une époque où les connaissances archéologiques de ces monuments demeuraient extrêmement réduites ${ }^{10}$.

Cependant l'idée que la villa doit se suffire à elle-même, qu'en bonne économie elle doit être construite et entretenue avec les moyens fournis par le domaine qui l'entoure, n'est pas totalement oubliée dans la littérature antique, notamment chez les poètes "bucoliques» comme Horace et Virgile, entraînant des confusions bien après eux sur le côté «naturel» de la villa. L'idéologie de la villa reste donc ambiguë, insistant sur les bienfaits de la campagne, de la vie aux champs, mais en se gardant bien de la mettre, sauf cliché poétique, au centre du dispositif.

Si l'on exclut la fonction économique de bonne gestion du domaine (agricole), le séjour dans une villa revêt à travers les âges plusieurs significations. La première, unanimement soulignée, concerne l'éloignement de la ville, et par là-même de tous ses effets négatifs, comme l'indiquent clairement les poètes. Mais derrière cette unanimité se cachent des aspirations contradictoires. La ville est globalement mauvaise et cela renvoie tout aussi bien aux conditions d'hygiène dues à l'entassement de la population qu'aux vices attribués à cette population (oisiveté, dévergondage, sans oublier la menace insurrectionnelle représentée par la «populace»). En opposition la vie à la campagne et donc dans une villa signifie le retour vers la nature et plus généralement celui de l'Âge d'Or, voire se traduit par la notion de "paradis ${ }^{11}$ sans référence à la vision chrétienne. Il en résulte la recherche privilégiée de lieux agréables et pittoresques, symbolisés par la notion d'amoenitas: un locus amoenus, un lieu plaisant, est indispensable pour l'établissement d'une villa résidentielle conduisant immédiatement à des distinctions sévères entre les secteurs ou les régions les plus aptes à recevoir ces villas et les autres. Cela apparaît clairement à propos des villas littorales où les côtes rocheuses demeurent nettement plus appréciées que les littoraux sableux ${ }^{12}$. En revanche, la pression démographique, à commencer par celle que l'on connaît à Rome, peut conduire à occuper des

9. Pline le Jeune, Lettres, II, 17, 13.

10. Les seuls monuments cités encore au XVIII ${ }^{\mathrm{e}}$ siècle se comptent sur les doigts de la main: Villa Adriana, villas d'Albano, Villas d'Anzio. Cf. Robert Castell, The villas of the Ancients, London, s. e, 1728.

11. Chez les aristocrates romains, qui ont emprunté cette notion aux civilisations procheorientales, le mot est régulièrement utilisé pour désigner un monde perdu.

12. C'est une différence importante avec les goûts de l'époque moderne où, notamment en Angleterre, on préfère les grandes plages de sable, plus favorables à la promenade comme au sport: sur ce point voir Alain Corbin, le Territoire du vide, L'Occident et le désir de rivage (17501840), Paris, Aubier, 1988, p. 84, 94. 
secteurs moins prisés, obligeant les architectes à d'autres types de prouesses techniques pour adapter un site a priori peu favorable à un degré élevé d'exigences. Pour en rester aux côtes sableuses et à un seul exemple, la présence de tours-belvédères permet de compenser la platitude du terrain naturel afin de favoriser une vision panoramique.

Pour en revenir au goût pour la nature campagnarde, les poètes augustéens comme Horace et Virgile ont largement développé ce point de vue et il est inutile d'insister davantage sur cet aspect ${ }^{13}$. On ne relève pas moins dans ce souci de fuir la ville plus d'une contradiction. La plus évidente tient dans le désir parallèlement affirmé de maintenir à la campagne un confort et une "qualité de vie» typiquement urbains. De ce point de vue, le choix de l'adjectif urbanus pour caractériser ces villas se passe de commentaire, mais on mentionnera particulièrement l'importance du balneum comme lieu de confort comme de sociabilité. L'hypertrophie de cet équipement dans certaines villas plus ou moins éloignées de la ville, leur accès indépendant, montrent qu'il n'était pas seulement destiné à l'usage du maitre et de sa famille, mais ouvert à une population plus large qui, vraisemblablement, ne se limitait pas aux seuls employés du domaine. À côté des bains hygiéniques matérialisés par les balnea, on relève l'existence de très nombreuses installations thermales, au sens moderne du mot, où l'on va pour se soigner loin du mauvais air de la ville. L'abondance des eaux thermales à proximité de Rome, mais également en Campanie, a donc largement favorisé le développement des villas résidentielles mais aussi, malgré cette abondance, leur concentration comme nous aurons l'occasion de le voir avec Baïes, "reine des stations thermales» antiques et lieu de villégiature par excellence.

La deuxième contradiction repose sur l'idée de "retraite», secessus en latin : on va dans une villa pour s'isoler, "faire retraite» loin de la foule et des contraintes sociales qu'elle impose. De ce fait, la plupart des grandes villas résidentielles comportent, à côté des salles de réception indispensables par ailleurs, des lieux plus isolés. Nouveau paradoxe, ces lieux peuvent également servir à recevoir un groupe plus ou moins importants d'invités. Le symbole de cet état de fait est donné par l'Amaltheum, le sanctuaire d'Amalthée, que fait ménager Cicéron dans sa villa de Tusculum. Il suffit de rappeler que dans la mythologie la chèvre (nymphe) Amalthée a recueilli le jeune Zeus pour le cacher dans une grotte et le dérober ainsi à la furie de son père Chronos qui voulait le tuer dès sa naissance de peur que, plus tard, il ne lui prenne son trône. Il s'agit donc de recréer dans la villa un lieu

13. Jean-Noël Robert, La vie à la campagne dans l'Antique Rome, Paris, Les Belles Lettres, 1985, p. 27-63 («Le mythe de la campagne»). 
16 / Histoire urbaine - 41 / décembre 2014

particulièrement fermé et isolé (une grotte en l'occurrence) où l'on pourra trouver refuge ${ }^{14}$. Dans le contexte particulièrement troublé des guerres civiles, où les exécutions sommaires des adversaires sont monnaie courante $^{15}$, la symbolique de ce lieu de retraite ne pouvait être que très forte comme l'a très bien montré Gilles Sauron ${ }^{16}$.

Cette idée de retraite à l'intérieur de la retraite que constitue en soi la villa a donné naissance à des constructions particulières connues tant par la littérature (outre l'Amaltheum de Cicéron, la tour et le "pavillon " de Pline dans sa villa des Laurentes) que par les restes archéologiques (le "théâtre maritime» de Villa Adriana, une micro villa installée au centre d'un bassin que l'on rejoint par un pont-levis). Mais cela peut être également toute la villa qui est aménagée dans cette perspective de retrait du monde. Le cas de la villa des Papyri à Herculanum est de ce point de vue exemplaire. L'ensemble du décor (la plus belle collection de statues en bronze et en marbre connue pour l'Antiquité) permettait à son propriétaire de se sentir hors du temps, plus précisément dans une Athènes mythique où l'épicurisme prit sa source à la fin du $\mathrm{III}^{\mathrm{e}}$ siècle. Ce "paradis" ainsi reconstitué apparaît sous des formes diverses, à commencer par l'Académie que Cicéron entend également aménager dans sa villa de Tusculum. La villa peut donc représenter une forme recherchée de nostalgie du temps passé, que l'on retourne aux temps homériques comme Tibère dans sa grotte de Sperlonga ${ }^{17}$ ou dans l'Athènes des philosophes pour Cicéron, le propriétaire de la villa des Papyri ou encore le contemporain de Pline le Jeune ${ }^{18}$. Cette liberté laissée au commanditaire de programmer sa villa selon ses goûts personnels, pour y réaliser ces aménagements très personnels, contraste fortement avec la domus urbaine qui doit respecter des règles de composition précises pour permettre au propriétaire d'y jouer son rôle politique ${ }^{19}$.

14. Henri Lavagne, Operosa antra, Recherches sur la grotte à Rome de Sylla à Hadrien, Rome, EFR, 1988, (BEFAR 272), particulièrement p. 257-263.

15. Il suffit de rappeler que les deux grands adversaires, Cicéron et Clodius, sont morts assassinés, le premier dans sa villa de Formies, le second en sortant de sa villa des monts Albains.

16. Gilles Sauron, «De Buthrote à Sperlonga: à propos d'une étude récente sur le thème de la grotte dans les décors romains", Revue Archéologique, 1991, p. 5-9 et Idem, "Un Amaltheum dans la villa d'Oplontis / Torre Annunziata ", Rivista di Studi Pompeiani, n ${ }^{\circ}$ 18, 2007, p. 41-46.

17. Bernard Andreae, Praetorium Speluncae-L'antro di Tiberio a Sperlonga ed Ovidio, Saveria Manelli, Rubbettino,1995 (Antiqua e Nova-Sezione Archeologia, IV).

18. Pline le Jeune, Lettres, VII, 25, 4 ( "Il pense vivre à Athènes plutôt que dans une villa »). Cf. John H. D'Arms, Commerce and social standing in Ancient Rome, London Harvard U. P. 1981, p. 11 .

19. D'après Vitruve, De Architectura, VI, 5, 1-2. Cf. Filippo Coarelli, «La casa dell'aristocrazia romana secondo Vitruvio «dans H Geertmann et Jakob Jan De Jong (edit.), Munus non ingratum, Proceedings of the International Symposium on Vitruvius De Architectura and the Hellenistic and Republican Architecture, Leyden, BABESCH, 1989, p. 178-187. 
Ce repli sur soi, dans le temps et dans l'espace, ne doit pas cependant être pris au pied de la lettre: l'aristocrate romain n'est pas un ascète se retirant seul au désert! Le propriétaire a toujours besoin d'une domesticité nombreuse, bruyante par définition ${ }^{20}$; surtout, il ne peut rester longtemps isolé de ses pairs. La villa est donc également un lieu de rencontres et de vie sociale complexe. Cela explique qu'à côté de villas qui aujourd'hui peuvent paraître très isolées les unes des autres car dispersées sur l'ensemble du territoire, on rencontre bien plus fréquemment des regroupements géographiques, de fait de véritables concentrations, qui concernent prioritairement les grandes villas aristocratiques. Quand l'espace reste malgré ce souci de proximité une contrainte seulement relative, la concentration n'interdit pas l'existence d'un domaine autour de chacune des villas (parcs d'agrément plus que champs cultivés cependant...). C’est le cas de la plupart des villes du suburbium romain, à commencer par celles qui occupent les sommets des Monts Albins (Alba, aujourd'hui Castel Gandolfo, Tusculum, etc.) ou les pentes des Monts Sabins (Tibur-Tivoli et Palestrina) ${ }^{21}$. Malgré une relative dispersion, les distances à parcourir pour se rendre d'une villa à l'autre restent faibles, de l'ordre de quelques kilomètres, parfois moins : à Tivoli, sur une surface de $40 \mathrm{~km}$ environ, on compte 54 villas mais avec des zones de plus grande concentration où l'on compte plusieurs villas au $\mathrm{km}$.

Dans le cas des sites littoraux, la proximité du rivage ainsi que la vue sur le large constituent une contrainte supplémentaire de localisation, si bien que l'on aboutit à une disposition des villas en complète contradiction avec le souci d'indépendance et d'isolement théoriquement recherché dans la villégiature... C'est le cas de Baies, dans le golfe de Naples, où dès l'époque augustéenne, Strabon reconnaissait que cette chaîne ininterrompue de villas ressemblait, de fait, à une ville ${ }^{22}$. Dans cette zone très recherchée, on assiste à des phénomènes de rejet des nouveaux venus par les «anciens", comme l'illustre le conflit qui sur ce point aussi oppose Cicéron et Clodius $^{23}$. Inutile d'insister sur le fait que Baïs apparaît égale-

20. Pline le Jeune, Lettres, II, 17, 24. Il évoque les fêtes des Saturnales particulièrement bruyantes, sorte de carnaval, qui se déroulent en janvier dans la villa.

21. Carte des villas du territoire de Tivoli dans Martin Tombrägel, Die republikanischen Otiumvillen von Tivoli, Wiesbaden, Reichert, 2012, (Pallila 25), fig. 1-3, p. 17-18.

22. Strabon, Géographie, V, 4, 7: «à Baïes s'est créée véritablement une seconde ville, aussi grande que Naples, depuis qu'on y construit des palais les uns après les autres" (traduction François Lasserre, CUF, 1967). Sur cette confusion entre villas littorales et villes, je me permets de renvoyer à Xavier Lafon, "Baies, une nouvelle forme de thermalisme ( $\mathrm{II}^{\mathrm{e}}-\mathrm{I}^{\mathrm{er}}$ siècles av. J.-C.)» dans Dominique Jarassé (édité par), 2000 ans de thermalisme, Économie, patrimoine, rites et pratiques, colloque de Royat (mars 1994), Clermont-Ferrand, IÉMC,1996, p. 11-22.

23. Xavier Lafon, Villa maritima, op. cit., p. 188 (avec les références à Cicéron). 
ment, dans la littérature latine du $\mathrm{I}^{\mathrm{er}}$ siècle av. J.-C. et dans celle du siècle suivant, comme "l'auberge de tous les vices " ${ }^{24}$, à commencer par la luxure, à l'égal de Rome elle-même, ou même en pire: il n'est donc pas loisible d'y venir chercher le repos et le calme comme le reconnait luimême le stoïcien Sénèque, qui y passe cependant de longs mois dans les dernières années de sa vie: en témoignent Les Lettres à Lucilius écrites entre 63 et 65 . Il est vrai qu'à cette date il a demandé officiellement sa retraite comme sénateur et "ami du Prince » ${ }^{25}$ et ses obligations sociales et politiques sont donc plus réduites que pour ses collègues. On aborde là une nouvelle série de contradictions à propos de la villégiature: est-elle légitime politiquement?

En dehors de la situation particulière qui est celle de Sénèque à la fin de sa vie, les rapports entre villa et activité politique conduisent en effet à des compromis qui, à l'intérieur des classes dirigeantes, témoignent d'une très grande diversité d'attitudes. Tout le monde s'accorde sur le fait que le secessus correspond fondamentalement à un véritable retrait de la vie politique, inadmissible dans son principe même pour le système romain, puisqu'il interdit de participer directement aux affaires publiques dans le cadre des deux institutions publiques majeures que sont le Sénat et le Forum, c'est-à-dire l'assemblée du populus et l'aide (juridique) apportée aux clients: tout cela implique nécessairement de se trouver dans la Ville, à Rome même. À l'échelle locale, on retrouve la même difficulté qui se traduit dans les lois municipales. Celles-ci obligent les hommes politiques, en l'occurrence les décurions, à posséder en tout état de cause une résidence urbaine intra muros $^{26}$, ce que l'on interprète comme le moyen de leur faire respecter leurs engagements politiques. Si l'on en revient aux sénateurs, le seul accommodement théorique envisageable est de réduire la durée de la villégiature aux périodes de vacances des activités politiques et judiciaires, c'est-à-dire aux deux mois d'été ${ }^{27}$. Indirectement, ce calendrier resserré permet de se retrouver dans les zones de villégiature, sans solution de continuité avec la vie sociale que l'on pratique à Rome...

24. Sénèque, Lettres à Lucilius, V, 51 .

25. Paul Veyne, Introduction à Sénèque-Entretiens, lettres à Lucilius, Paris, 1993, (Collection Bouquins), p. XXXV et p. CLVII, citant la Lettre 73,4: «Mais voici quelqu'un, cœur net et candide, qui a quitté le Sénat, le forum, toute participation au gouvernement pour se consacrer dans l'isolement à une tâche plus vaste».

26. La résidence urbaine n'est pas une donnée aussi «naturelle» que ce que l'on pense généralement dans le monde de la Cité et ces rappels à l'ordre législatifs montrent que la tendance à préférer vivre à la campagne ne concernait pas uniquement les aristocrates de Rome préférant l'otium (le loisir dans la retraite) au negotium (l'activité, à commencer par l'activité politique).

27. Depuis que les campagnes militaires ne concernent plus qu'un petit nombre d'aristocrates en raison d'une armée de métier. 
Une deuxième forme d'accommodement possible résulte d'une proximité immédiate de la villa par rapport à la Ville, situation qui autorise l'activité au forum le matin et un retour dans la villa dès la fin de l'aprèsmidi $^{28}$. On retrouve à ce propos toute la complexité, mais également l'élasticité, de la notion de suburbium, avec la catégorie particulière des villas qualifiées de suburbaines. Au sens strict, le suburbium commence dès que l'on a franchi la muraille urbaine et la bande du pomoerium. La situation se complique quand il s'agit de fixer la limite externe du suburbium! J'avais proposé ${ }^{29}$ de prendre en compte une ligne isochrone correspondant à une journée de cheval, soit un cercle autour de Rome d'environ $50 \mathrm{~km}$, permettant d'intégrer plusieurs grandes villas impériales à commencer par Anzio (où est né Néron), Tivoli, Albano etc., mais en aucun cas les sites les plus recherchés de la Campanie comme Baïes. Le cas limite pourrait être Civitavecchia (Centumcellae) où l'empereur Trajan réunit son conseil et poursuit donc son activité "normale», comme s'il était à Rome même. Au-delà, les délais de route sont trop longs et rendent nécessaires des séjours de plus longue durée. En revanche une villa dans le suburbium permet des séjours tout au long de l'année, rompant à l'époque impériale avec le calendrier resserré de l'été ${ }^{30}$.

Dans tous les cas envisagés jusqu'à présent, le séjour dans la villa n'est pas en contradiction avec les devoirs d'un sénateur (voire de l'empereur) soit que le séjour se place en période de vacances soit qu'il se déroule à proximité même de Rome, autorisant des allers-retours réguliers ou un retour dans l'urgence comme celui de Néron apprenant à Anzio l'incendie de Rome, avec la possibilité d'assister dans les heures qui suivent au spectacle du haut de la Tour de Mécène ${ }^{31}$.

À côté des ces arrangements, il existe des cas où la rupture apparaît recherchée de façon en quelque sorte provocatrice. Le phénomène apparaît caractéristique de la fin de l'époque républicaine ( $\mathrm{I}^{\mathrm{er}}$ siècle av. J.-C.) dans le milieu des vieilles familles aristocratiques accusé par le "nouveau venu» Cicéron de préférer leurs viviers à poissons (dans des villas de Campanie tout particulièrement) à la défense de la patrie et de ses institutions ${ }^{32}$.

28. Pline le Jeune, Lettres, II, 17, 1 ("À 17 milles de Rome [la villa] a trouvé une retraite [secessit] telle qu'on puisse, une fois quitte de ses occupations, sans entamer ni écourter sa journée de travail, venir y passer la nuit». Traduction A.-M. Guillemin, CUF.

29. Xavier Lafon, Villa Maritima, op. cit., p. 130.

30. Cet élargissement de la période où la villa peut accueillir le propriétaire est à mettre en rapport avec des progrès techniques dans la construction, notamment l'apparition du verre à vitre au ${ }^{\text {er }}$ siècle après J.-C., indispensable pour un séjour hivernal dans une villa littorale.

31. Suétone, Vie de Néron, 38; Tacite, Annales, XV, 38-41.

32. Xavier Lafon, Villa Maritima, op. cit., p. 198. 
Au-delà de la polémique que l'on interprète trop souvent au premier degré (le désintérêt pour la chose publique), cette situation me semble traduire une véritable alternative au schéma du fonctionnement de la cité, reposant en partie au moins sur un séjour loin de la capitale. La traduction la plus achevée de ce comportement est certes postérieure, incarnée dans la littérature antique par l'empereur Tibère se retirant à Capri où il réside pendant les 14 dernières années de son règne, sans remettre les pieds une seule fois à Rome ${ }^{33}$. Cette attitude est considérée par les auteurs d'esprit républicain, proches du point de vue et des intérêts sénatoriaux, à commencer par Tacite et Suétone, comme proprement tyrannique, au sens où l'empereur ne réunit plus les organes habituels ni ne remplace les officiers et les magistrats. Il s'agit incontestablement d'une politique de rupture, vraisemblablement inaugurée par Sylla plus d'un siècle auparavant quand, nommé dictateur, il se retire en Campanie ${ }^{34}$. En raison d'une mort précoce, ce dernier n'eut toutefois pas le temps de la mettre véritablement en application, mais ses héritiers spirituels figurent parmi les membres de la nobilitas que dénonce Cicéron parmi les amateurs de viviers et ce n'est certainement pas un hasard... De fait, malgré les allégations des auteurs antiques, Tibère continue bien de gouverner l'Empire à l'aide de bureaux transplantés à Capri, grâce à la flotte et à une série de sémaphores ${ }^{35}$ qui lui permettent de se tenir au courant et de transmettre les ordres. Mais un tel schéma n'était pas véritablement concevable et acceptable dans la mentalité des auteurs latins dont nous connaissons les œuvres: on doit gouverner depuis la capitale et donc y résider normalement, la villégiature n'étant qu'un intermède, pas une fin en soi.

Mais ce n'est pas seulement le chef de l'État qui entend mener une "autre politique», retiré dans une de ses villas plus ou moins éloignée de Rome mais pourvue des moyens bureaucratiques et techniques nécessaires pour administrer l'Empire. Les chefs des grandes familles, ancienne nobilitas ou nouveaux venus, ont besoin à la fin de la République de clients en grand nombre, mais les plus intéressants dans un système censitaire appartiennent aux classes supérieures et donc à la catégorie des possesseurs de villas ${ }^{36}$. Le réseau ainsi constitué peut dépasser pour certains imperatores comme Pompée ou César le cadre de l'Italie quand ils jouent sur les différentes charges exercées dans les provinces. Mais il

33. Suétone, Vie de Tibère, 39 et 72.

34. Appien, Guerres civiles, $1,104$.

35. Suétone, Vie de Tibère, 65.

36. Le système censitaire permettait de gagner des élections avec finalement très peu de votants comparé à la masse des citoyens romains. Claude Nicolet, Le métier de citoyen dans la Rome républicaine, Paris, Gallimard, 1976, p. 280-418, particulièrement p. 375. 
existe parallèlement, sinon antérieurement, une dimension péninsulaire, marquée pour l'homme politique par la possession de villas, généralement dans la cité d'origine (origo) de la famille. Grâce à l'abondance et aux spécificités de sa Correspondance, Cicéron nous en livre un exemple type, avec son regnum (le mot n'est pas neutre et ne peut apparaître que dans une correspondance privée) organisé avec son frère Quintus autour d'Arpinum. Cette présence (Cicéron y fait de nombreux séjours même s'ils sont brefs), les achats et les travaux entrepris en particulier dans sa villa principale héritée de son père, ne sont pas uniquement liés à l'amour de la "petite patrie». Cette attention, même si elle n'est pas explicite, ne peut être sans rapport avec la place que Cicéron entend occuper sur l'échiquier politique romain. On comprend qu'il connaisse parallèlement toute la géographie des villas italiennes et de leurs propriétaires dans un marché qui, paradoxalement ne cesse d'évoluer, les propriétés rurales changeant régulièrement de mains.

Il est donc important pour un homme politique romain de se constituer des appuis dans une région qu'il connaît bien, mais ce souci concerne également les régions de grande villégiature comme le golfe de Naples. Cicéron se plaint ${ }^{37}$ de ne pas trouver la paix quand il séjourne dans ses villas de Formies ou de Cumes, où il exerce la même activité politique qu'à Rome, à commencer par la réception quotidienne d'un nombre important de clients. À cela s'ajoute, à proximité de ces villes portuaires à commencer par Pouzzoles, des opportunités économiques importantes, même si la codification imposée des activités interdit à un sénateur de se livrer directement au commerce: on dispose de nombreux indices qui tendent à montrer que Cicéron lui-même était largement engagé dans le commerce maritime et les activités manufacturières. La possession d'une villa et des séjours prolongés au moment de l'année où la navigation au long cours est la plus active constituent donc des atouts considérables pour réaliser, plus ou moins discrètement, des affaires.

Cet ancrage local, appuyé sur la possession de villas souvent éloignées de Rome, ne disparaît pas avec l'Empire même si le nouveau régime tend à le contrôler en rendant les déplacements plus difficiles: sauf pour la Sicile et la Narbonnaise, les sénateurs doivent obtenir une autorisation impériale pour se rendre dans leurs domaines. Au désir de l'Empereur d'avoir son monde à disposition pour administrer l'Empire a pu s'ajouter le désir d'éviter la constitution de "royaumes " susceptibles d'échapper à l'autorité centrale. Les raisons (positives) pour un aristocrate romain de se rendre et

37. Cicéron, Lettres à Atticus, V, 2 («nous avons à Cumes comme une petite Rome en raison d'une foule immense»). 
de résider dans ses villas ne manquaient donc pas, mais se heurtaient à des contraintes tout aussi fortes, de nature idéologique et politique. Les textes des auteurs antiques, dans leur variété, traduisent davantage ces tensions que les découvertes archéologiques. Ces dernières témoignent pourtant de la réalité concrète et de l'importance acquise par le phénomène de la villégiature antique. Ce n'est pas le moindre paradoxe que de voir que la lecture des premiers n'a pas interdit aux modernes de concevoir à leur tour des formes complexes de séjour hors des murs de la ville, bien avant l'essor des congés pour tous.

J'ai conscience, dans ce bref rappel de la villégiature aristocratique romaine, d'avoir présenté une vision nécessairement réductrice de la situation antique. Réductrice en premier lieu parce qu'elle concerne un nombre très réduit d'individus et de familles, quelques centaines tout au plus, à comparer aux dizaines de milliers d'établissements ruraux qualifiés de villas repérés dans l'ensemble du monde romain. Réductrice surtout parce qu'elle repose fondamentalement sur la vision d'elle-même qu'a voulu nous transmettre cette aristocratie, indifférente aux autres catégories sociales et à leurs préoccupations. En revanche, cette vision essentiellement littéraire est celle que les érudits de la Renaissance ont mise en lumière, offrant à leurs contemporains et aux siècles suivants un cadre, certes figé, dans lequel les commanditaires potentiels pouvaient puiser à loisir, avec la caution suprême apportée par l'antiquité de ces pratiques. Bien évidemment, les conditions socio-économiques de l'époque contemporaine voire déjà moderne, n'ont plus grand-chose à voir avec celles de la fin de la République ou du Haut-Empire romain et les modalités de réalisation de ces "nouvelles villas» ne sont pas directement comparables. La culture antique demeure cependant suffisamment présente pour que les descriptions de Pline, jusqu'à une date très récente ${ }^{38}$, servent d'exercice à de futurs architectes ${ }^{39}$, voire à des architectes confirmés, alors que leur caractère nécessairement incomplet ne peut plus être mis en doute. Plus largement encore, le souhait de quitter la ville pour la villégiature comporte de nos jours autant de contradictions que dans l'Antiquité romaine.

38. Un concours sur le thème de la restitution de la villa des Laurentes de Pline a été encore organisé en 1982 (La Laurentine, Catalogue de l'exposition, Institut français d'architecture, Paris, Éditions du Moniteur, 1982).

39. Vaudoyer, Le Laurentin, programme d'architecture proposé aux élèves de l'école royale d'architecture pour le mois de novembre 1818, Paris, cité dans Dominique Rouillard, Le site balnéaire, Bruxelles (Mardaga), 1984, en particulier chapitre 9: Pline et Michelet: «système des éléments ", p. 259-276. 